\title{
Edge-Directed Image Interpolation Using Color Gradient Information
}

\author{
Andrey Krylov and Andrey Nasonov* \\ Laboratory of Mathematical Methods of Image Processing, \\ Faculty of Computational Mathematics and Cybernetics, \\ Lomonosov Moscow State University, \\ 119991, Leninskie gory, Moscow, Russia \\ $\{k r y l$, nasonov\}@cs.msu.ru \\ http://imaging.cs.msu.ru/
}

\begin{abstract}
Image resampling method using color edge-directed interpolation has been developed. It uses color image gradient to perform the interpolation across image gradient rather than along image gradient. The developed combined method takes color low resolution image and grayscale high resolution image obtained by a non-linear image resampling method as an input. It includes consecutive calculation stages for high resolution color gradient, for high resolution color information interpolation and finally for high resolution color image assembling.

The concept of color basic edges is used to analyze the results of color image resampling. Color basic edge points metric was suggested and used to show the effectiveness of the proposed image interpolation method.
\end{abstract}

Keywords: color image upsampling, gradient based interpolation.

\section{Introduction}

Image interpolation is as a key part of many image processing algorithms. For example, image interpolation is performed when a low-resolution video is shown on a high-resolution display. Preserving edge information is the main problem of image interpolation algorithms.

Color image interpolation is generally used in image demosaicing [8] while most of existing approaches in color image upsampling are reduced to grayscale image interpolation by decomposing the image into individual color components and processing these components independently. There exists a large variety of edge-directed image interpolation algorithms [6]712, but most of them do not take into account color edges. If the image is converted to a color model with separated luminance (brightness) and chrominance (color) components like YUV, the attention is usually paid to image luminance only while chrominance components are interpolated using simple methods like bilinear or bicubic interpolation.

\footnotetext{
* The work was supported by Russian Foundation for Basic Research grant 10-0100535 .
} 
This approach does not lead to significant degradation of the perceptual image quality because the sensitivity of the human perception to the change of image intensity is higher than to the change of image color. But there is a small portion of edges which are strong in chrominance components and weak in the luminance component. In [4] it was shown that about 10 percent of image edges are not detected in the luminance component. Low quality of interpolation of these edges may be annoying.

In this paper, we present a method to improve the interpolation of chrominance components using multichannel gradient. The concept of color basic edges is used to find the edges where involving color information can significantly improve the quality.

\section{Gradient Based Grayscale Image Interpolation}

We use gradient based method for the interpolation of single component images. The idea of gradient methods is to use different interpolation kernels depending on image gradient 2]. We calculate the value of pixel being interpolated as a weighted sum of pixels laying along the normal to the gradient in the interpolated pixel.

In our work, we consider linear interpolation method [1]

$$
f(x, y)=\frac{\sum_{i, j} u_{i, j} K\left(\sqrt{(x-i)^{2}+(y-j)^{2}}\right)}{\sum_{i, j} K\left(\sqrt{(x-i)^{2}+(y-j)^{2}}\right)}
$$

where $u_{i, j}$ is the low-resolution image, $f(x, y)$ is the interpolated image, $K(t)$ is the interpolation kernel.

To construct the edge adaptive gradient image interpolation algorithm based on the linear interpolation method, we stretch the interpolation kernel in the normal direction to the image gradient:

$$
f(x, y)=\frac{\sum_{i, j} u_{i, j} K\left(\sqrt{\left(x^{\prime}\right)^{2}+\left(\frac{1}{\sigma_{x, y}} y^{\prime}\right)^{2}}\right)}{\sum_{i, j} K\left(\sqrt{\left(x^{\prime}\right)^{2}+\left(\frac{1}{\sigma_{x, y}} y^{\prime}\right)^{2}}\right)},
$$

where $x^{\prime}$ and $y^{\prime}$ are coordinates of points $(i, j)$ in the coordinate system centered in the point $(x, y)$ with the axe $O x$ directed along the gradient direction in the point $(x, y)$ :

$$
\begin{gathered}
x^{\prime}=(x-i) \cos \theta_{x, y}+(y-j) \sin \theta_{x, y}, \\
y^{\prime}=-(x-i) \sin \theta_{x, y}+(y-j) \cos \theta_{x, y} .
\end{gathered}
$$

The value $\theta_{x, y}$ is the angle of the gradient in the point $(x, y)$ and $\sigma_{x, y}$ is the kernel deformation coefficient which depends on the value of the gradient $\left|g_{x, y}\right|$. 
We use the following value

$$
\sigma_{x, y}= \begin{cases}\sigma_{0}, & \left|g_{x, y}\right| \geq g_{0}, \\ 1+\left(\sigma_{0}-1\right) \frac{\left|g_{x, y}\right|}{g_{0}}, & 0 \leq\left|g_{x, y}\right|<g_{0},\end{cases}
$$

where the values $\sigma_{0} \geq 1$ and $g_{0}>0$ are the parameters of the proposed gradient method. Using $\sigma_{0}=1$ will convert the gradient image interpolation method (2) to linear interpolation method (1). These parameters are specific to the interpolated image class and the interpolation kernel $K(t)$.

For the bicubic interpolation kernel

$$
K(t)= \begin{cases}(a+2)|t|^{3}-(a+3)|t|^{2}+1 & \text { for }|t| \leq 1 \\ a|t|^{3}-5 a|t|^{2}+8 a|t|-4 a & \text { for } 1<|t|<2 \\ 0 & \text { otherwise }\end{cases}
$$

with $a=-0.5$ and images from LIVE image database [1011] it was found that using the values $\sigma_{0}=1.5$ and $g_{0}=20$ maximizes the $S S I M$ metric value 13 .

Fig. 11 illustrates the proposed gradient image interpolation method.
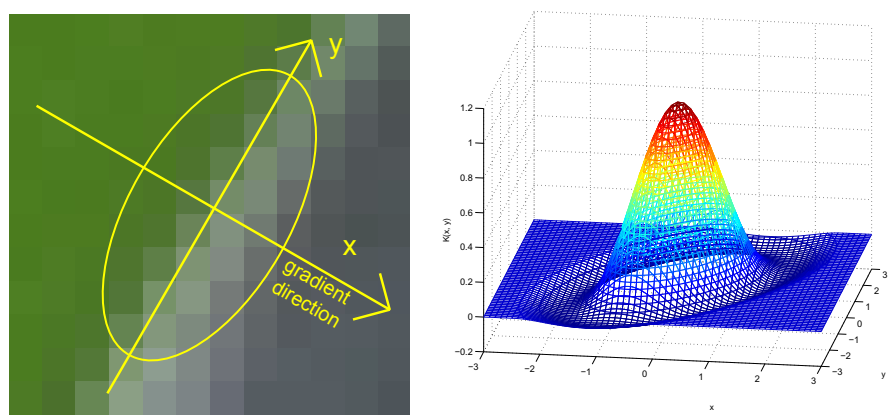

Fig. 1. The kernel function of the gradient based method in the point on the edge for $\sigma_{x_{0}, y_{0}}=2$

The proposed gradient based image interpolation method shows better results than linear methods but worse than high quality non-linear algorithms like NEDI [7] or algorithms based on regularization [5]. Nevertheless the proposed method is significantly faster than non-linear image resampling algorithms.

\section{Gradient Based Color Image Interpolation}

The idea of color image interpolation is to use the multichannel image gradient proposed by Di Zenzo 3. It finds the direction $\theta_{0}$ that maximizes the rate of change function

$$
F(\theta)=g_{x x} \cos ^{2} \theta+2 g_{x y} \sin \theta \cos \theta+g_{y y} \sin ^{2} \theta,
$$



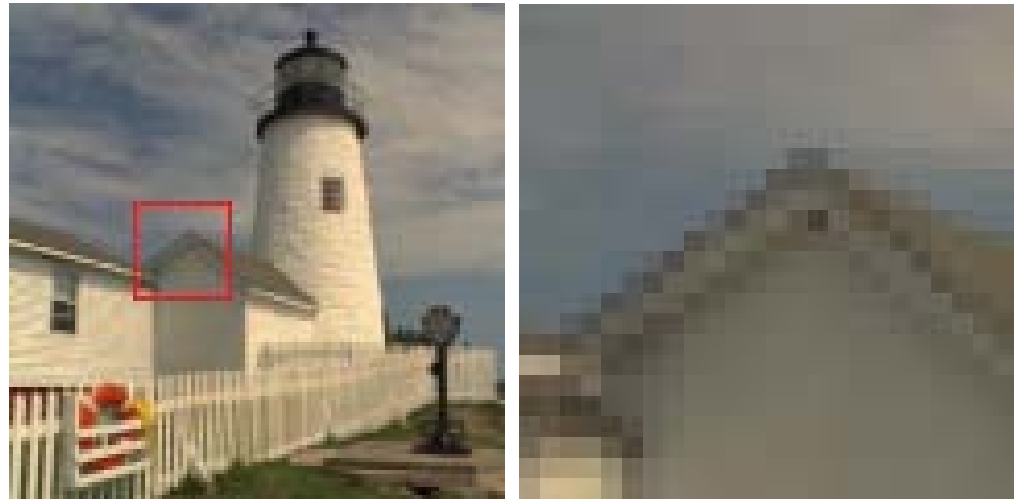

Low resolution image

Nearest neighbor, PSNR $=16.944$

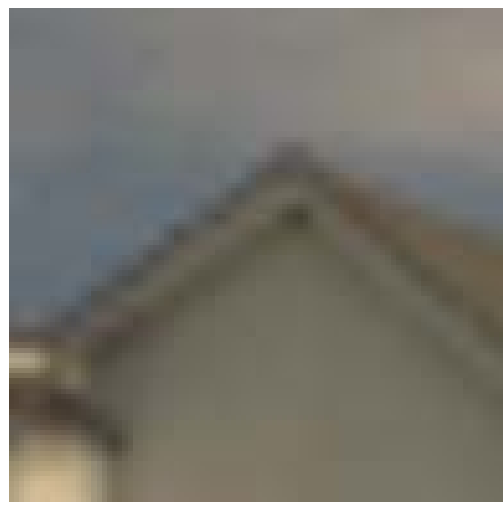

Bicubic interpolation, $\mathrm{PSNR}=18.630$

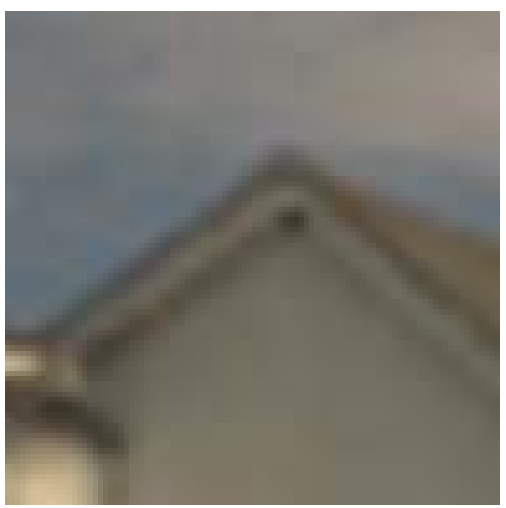

Color gradient based interpolation, $\mathrm{PSNR}=18.775$

Fig. 2. The results of gradient based 4 times image upsampling for color images with bicubic kernel function

where

$$
\begin{gathered}
g_{x x}=\left(\frac{\partial R}{\partial x}\right)^{2}+\left(\frac{\partial G}{\partial x}\right)^{2}+\left(\frac{\partial B}{\partial x}\right)^{2}, \\
g_{y y}=\left(\frac{\partial R}{\partial y}\right)^{2}+\left(\frac{\partial G}{\partial y}\right)^{2}+\left(\frac{\partial B}{\partial y}\right)^{2}, \\
g_{x y}=\frac{\partial R}{\partial x} \frac{\partial R}{\partial y}+\frac{\partial G}{\partial x} \frac{\partial G}{\partial y}+\frac{\partial B}{\partial x} \frac{\partial B}{\partial y} .
\end{gathered}
$$

The explicit formula to find the direction of the multichannel gradient $\theta_{0}$ looks as

$$
\theta_{0}=\frac{1}{2} \arctan \frac{2 g_{x y}}{g_{x x}-g_{y y}} .
$$

The value $F\left(\theta_{0}\right)$ is the gradient power. 
We apply the grayscale gradient image interpolation method (2) to color images using Di Zenzo gradient. Fig. 2 shows the results of gradient image interpolation with the bicubic kernel function.

\section{Improvement of Color Image Interpolation by the Gradient Based Method}

The proposed gradient method cannot give results with quality compared to high-quality non-linear methods even when color image gradient is used. But processing of all components of the color image by high-quality methods is time consuming.

We suggest the algorithm for color image interpolation which takes a color low resolution image and a grayscale high resolution image obtained by a highquality grayscale image interpolation method as input data. The grayscale highresolution image is used to refine the color image gradient. The algorithm consists of the following steps:

1. Calculate the color high-resolution image with chrominance components interpolated from the given low-resolution image using bicubic interpolation and the luminance component taken from the given grayscale high-resolution image.

2. Perform interpolation of the given low-resolution image by the proposed color gradient based method with color gradient taken from the color highresolution image obtained in the previous step.

3. Take chrominance components of the obtained in the previous step color image resolution image and add it to the given grayscale high resolution image. The obtained image is the result of the algorithm.

\section{Color Basic Edges}

To analyze the quality of the proposed algorithm, we seek for edges where the difference between gradient based interpolation and linear methods is noticeable.

For grayscale images, the concept of basic edges [9] is used to find the edges which can be used to estimate image quality. By the term 'basic edges' sharp edges distant from other edges are called. Blur and ringing effect are the most noticeable near these edges.

We extend the concept of basic edges to grayscale images using multichannel image gradient and color edge detection. But since human eye is more sensitive to intensity changes than to color changes, color artifacts are not visible if color basic edge is also a grayscale basic edge. Therefore, we seek for color basic edges which are not grayscale basic edges. We call these edges as pure color basic edges.

The example of color basic edges detection in shown in fig. 3. 


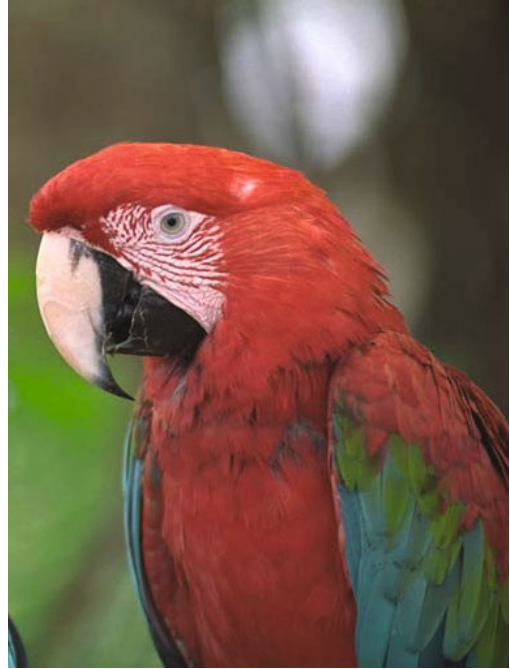

The reference image.

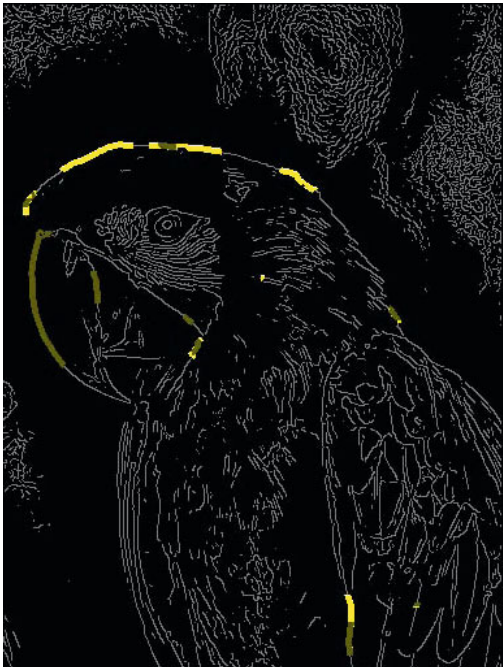

The result of basic edges detection.

Fig. 3. The results of finding basic edges areas for the image 'parrots'. Gray lines are image edges. Dark yellow area is the area of edges being both color and grayscale basic edges. Light yellow area is the area of color basic edges which are not detected as grayscale basic edges.

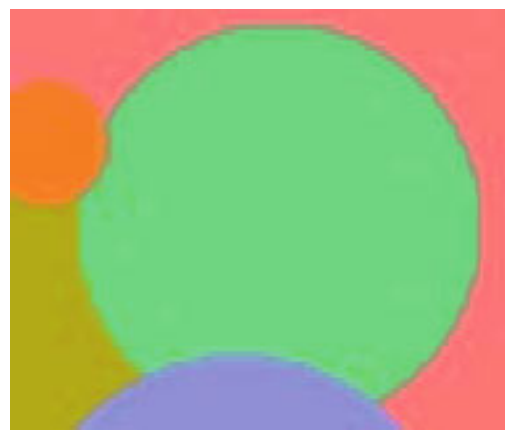

Bicubic interpolation

$\mathrm{CBEP}=7.93, \mathrm{PSNR}=24.616$

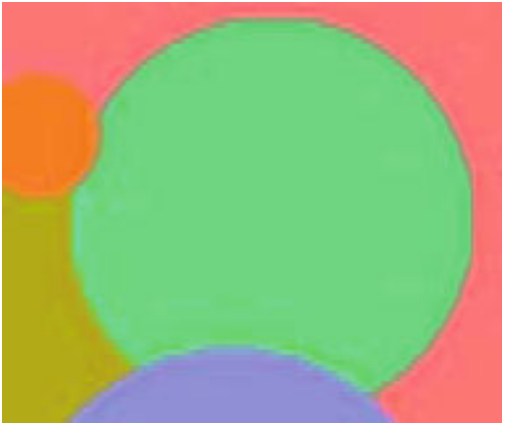

Gradient based interpolation $\mathrm{CBEP}=10.98, \mathrm{PSNR}=25.584$

Fig. 4. The results of the proposed interpolation methods for the synthetic image with color edges only

\section{Results}

To evaluate the proposed method we took the reference images, downsampled it in 4 times, then applied regularization based image interpolation [5] and the proposed methods and compared the results with the reference images. 


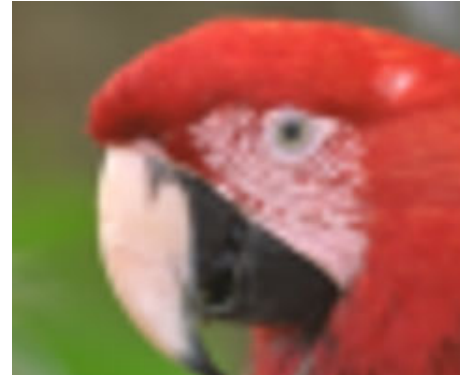

Bicubic interpolation

$\mathrm{CBEP}=32.26, \mathrm{PSNR}=24.433$

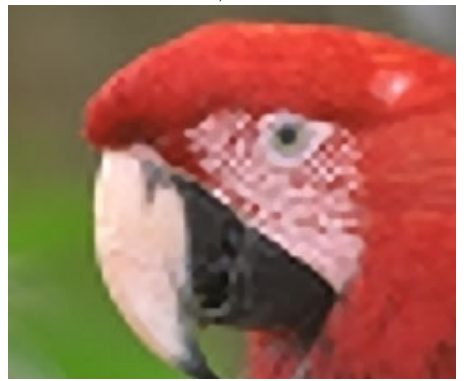

Regularization based interpolation [5] with bicubic interpolated chrominance components $\mathrm{CBEP}=45.45, \mathrm{PSNR}=25.038$

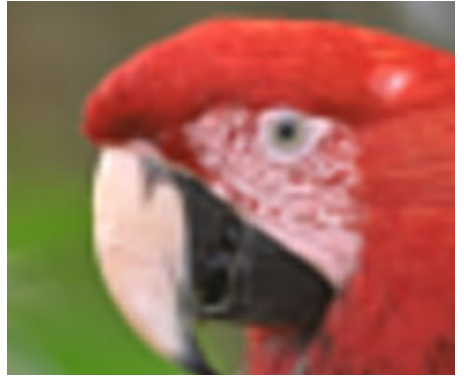

Gradient based interpolation $\mathrm{CBEP}=41.66, \mathrm{PSNR}=24.586$

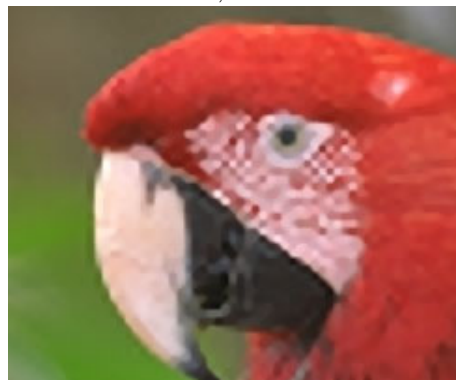

Regularization based interpolation with gradient based interpolated chrominance components $\mathrm{CBEP}=52.63, \mathrm{PSNR}=25.077$

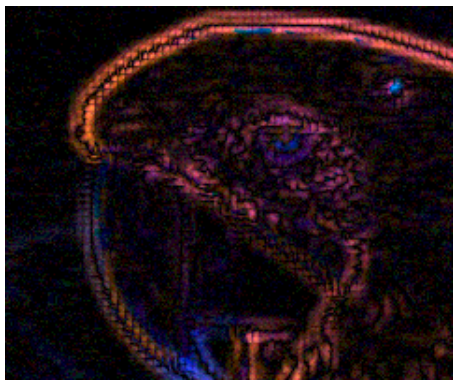

The difference image between the last two images (32 times amplified)

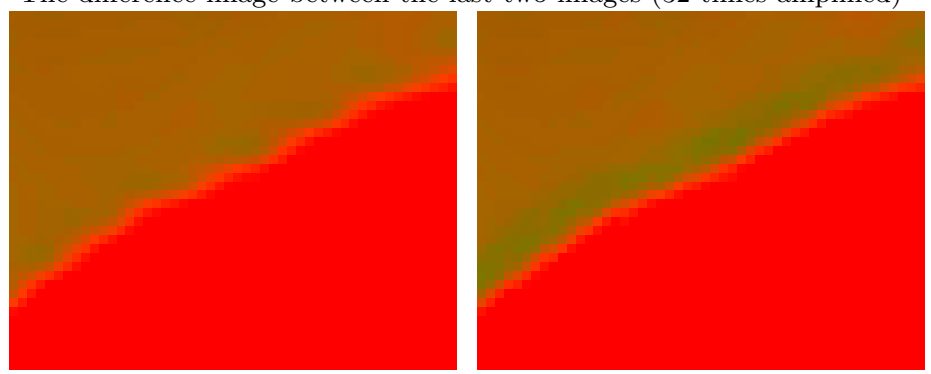

Amplified image fragments of the last two images

Fig. 5. Application of the proposed color image interpolation methods to the image 'parrots' 


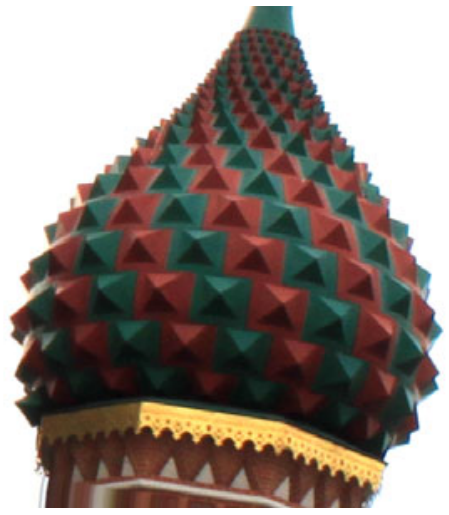

Reference image

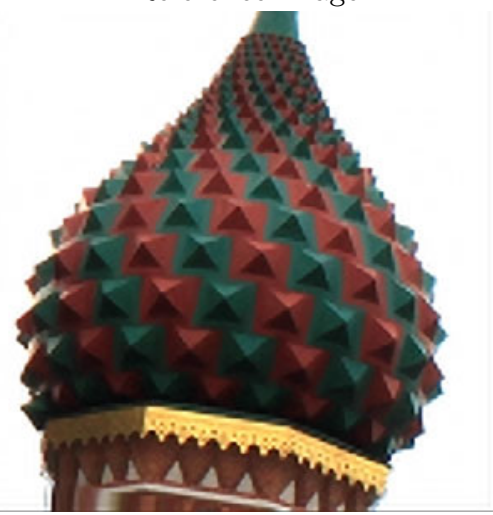

Regularization based interpolation with bilinear interpolated chrominance components $\mathrm{CBEP}=43.47, \mathrm{PSNR}=25.585$

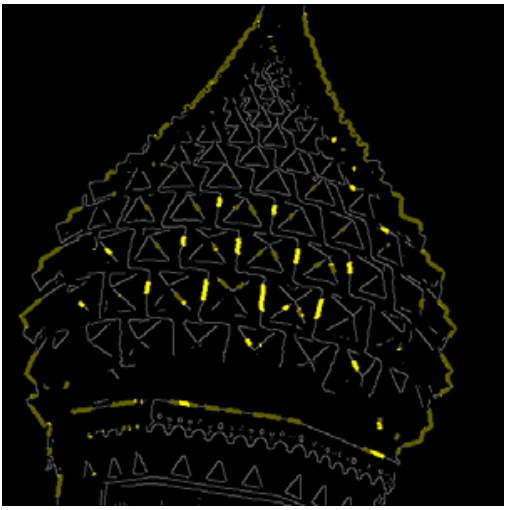

Basic edges

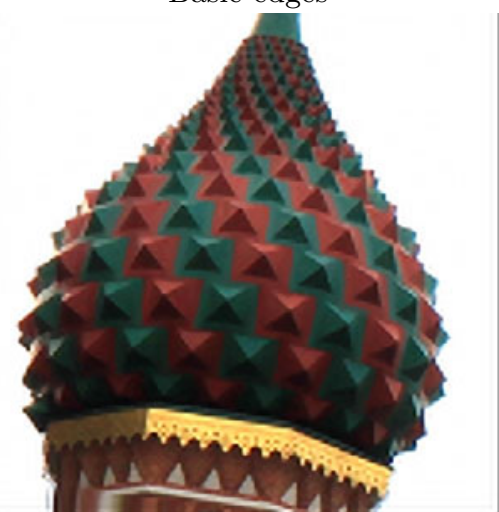

Regularization based interpolation with gradient based interpolated chrominance components $\mathrm{CBEP}=50.00, \mathrm{PSNR}=25.613$
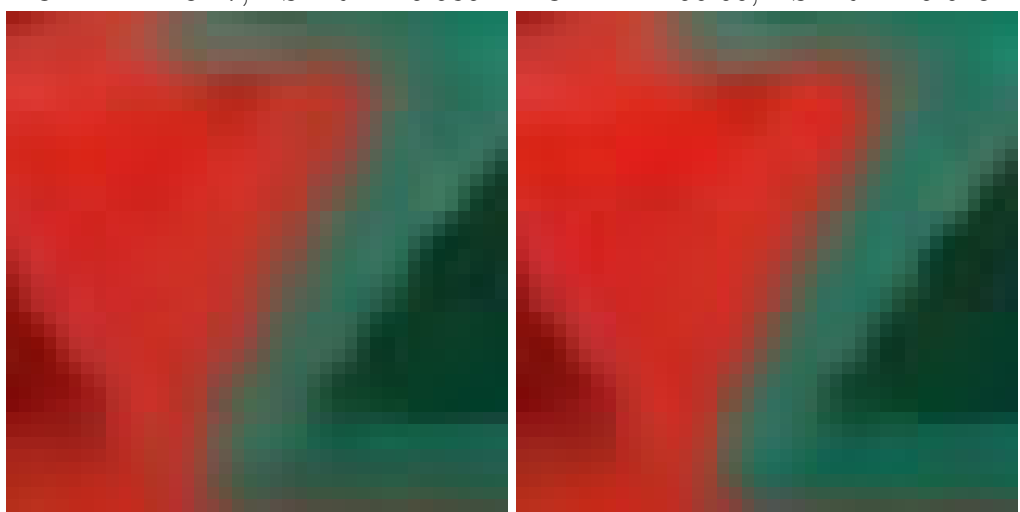

Amplified image fragments of the last two images

Fig. 6. Application of the proposed color image interpolation methods to the image 'cathedral' 
Two metrics were calculated: PSNR and CBEP. The metric CBEP equals to DSSIM [13] metric calculated only in the area of interest $M_{C B E P}$. This area is the analog to BEP area introduced in [9] for color images. It consists of pixels in a small neighborhood of pure color basic edges. The metric PSNR was calculated for the entire image.

The results of the proposed interpolation methods for the synthetic image with only pure color basic edges are shown in Fig. 4. It can be seen that the gradient based method with bicubic kernel shows better edge quality than bicubic interpolation method.

For real images, the difference is less noticeable because of noise and few edges with completely absent grayscale gradient. In fig. 5 the results of the proposed color image interpolation methods for the image 'parrots' are shown. If bilinear interpolation is used for chrominance components, the difference is more visible. In fig. 6 the results for the image 'cathedral' with bilinear interpolation of color components are shown.

\section{Conclusion}

The gradient method for color image interpolation has been proposed. It uses the multichannel gradient to perform high quality interpolation of color edges that are not presented in the luminance image component.

The proposed algorithm enhances the grayscale high resolution image obtained by a non-linear image resampling method with bilinear or bicubic chrominance components resampling. The effect can be seen in the area of image color basic edges. This approach can be used to improve the performance of high quality but very slow image interpolation algorithms that process all components of the image. Instead of processing all components of the image, it is possible to resample only luminance component by high quality algorithm and to interpolate chrominance components by the proposed gradient method.

\section{References}

1. Blu, T., Thevenaz, P., Unser, M.: Linear interpolation revitalized. IEEE Trans. Image Proc. 13(5), 710-719 (2004)

2. Chena, M.J., Huanga, C.H., Leea, W.L.: A fast edge-oriented algorithm for image interpolation. Image and Vision Computing 23(9), 791-798 (2005)

3. Di Zenzo, S.: A note on the gradient of a multi-image. Comput. Vision Graph. Image Process. 33, 116-125 (1986)

4. Koschan, A.: A comparative study on color edge detection. In: Li, S., Teoh, E.-K., Mital, D., Wang, H. (eds.) ACCV 1995. LNCS, vol. 1035, pp. 574-578. Springer, Heidelberg (1996)

5. Krylov, A.S., Lukin, A.S., Nasonov, A.V.: Edge-preserving nonlinear iterative image resampling method. In: Proceedings of International Conference on Image Processing (ICIP 2009), pp. 385-388 (2009)

6. Lee, Y.J., Yoon, J.: Nonlinear image upsampling method based on radial basis function interpolation. IEEE Trans. Image Proc. 19(10), 2682-2692 (2010) 
7. Leitao, J.A., Zhao, M., de Haan, G.: Content-adaptive video up-scaling for highdefinition displays. In: Proceedings of Image and Video Communications and Processing 2003, vol. 5022, pp. 612-622 (2003)

8. Li, X., Gunturk, B., Zhang, L.: Image demosaicing: a systematic survey. Visual Communications and Image Processing 6822, 68221J-68221J-15 (2008)

9. Nasonov, A.V., Krylov, A.S.: Basic edges metrics for image deblurring. In: Proceedings of 10th Conference on Pattern Recognition and Image Analysis: New Information Technologies, vol. 1, pp. 243-246 (2010)

10. Sheikh, H., Sabir, M., Bovik, A.: A statistical evaluation of recent full reference image quality assessment algorithms. IEEE Trans. Image Proc. 15(11), 3440-3451 (2006)

11. Sheikh, H., Wang, Z., Cormack, L., Bovik, A.: Live image quality assessment database release 2 , http://live.ece.utexas.edu/research/quality

12. Sun, J., Xu, Z., Shum, H.Y.: Image super-resolution using gradient profile prior. In: IEEE Conf. on Computer Vision and Pattern Recognition (CVPR 2008), pp. 1-8 (2008)

13. Wang, Z., Bovik, A., Sheikh, H., Simoncelli, E.: Image quality assessment: from error visibility to structural similarity. IEEE Trans. Image Proc. 13(4), 600-612 (2004) 